\title{
Force isolation by locally resonant metamaterials to reduce NVH
}

\author{
Author, co-author (Do NOT enter this information. It will be pulled from participant tab in \\ MyTechZone) \\ Affiliation (Do NOT enter this information. It will be pulled from participant tab in MyTechZone)
}

\begin{abstract}
The combination of lightweight design and performant Noise, Vibrations Harshness (NVH) solutions has gained a lot of importance over the past decades. Lightweight design complies with the ever more stringent environmental requirements, however conflicts with $\mathrm{NVH}$ performance, as low noise and vibration levels often require heavy and bulky systems, especially at low frequencies. To face this challenge, locally resonant metamaterials come to the fore as low mass, compact volume $\mathrm{NVH}$ solutions, beating the mass law in some tunable frequency zones, referred to as stopbands. Metamaterials are artificial materials made from assemblies of unit cells of non-homogeneous material composition and/or topology. The local interaction between unit cells leads to superior performance in terms of noise and vibration reduction with respect to the conventional NVH treatments. Previously the authors showed how wave propagation along one-dimensional structures can be reduced by metamaterial additions. In this paper the authors apply the concept of metamaterials to reduce vibration in a complex 2D structure, excited at one specific input location. Numerical and experimental results are shown for a simplified set-up representing part of a body of a vehicle excited by a shock absorber. By adding metamaterials on a limited portion of the structure, an average of $6.8 \mathrm{~dB}$ with a minimum of $3 \mathrm{~dB}$ vibration reduction is achieved in a $50 \mathrm{~Hz}$ frequency band centered around the target frequency of $300 \mathrm{~Hz}$, by only adding 2.4 percent of mass to the structure. The frequency band, the attenuation and the added mass are all tunable by design.
\end{abstract}

\section{Introduction}

Lightweight design solutions are increasingly used for industrial applications, especially in automotive, due to economic and ecologic requirements. Performant NVH solutions are needed to provide an optimal acoustic comfort and to limit the impact of noise exposure on human health. Lightweight design, characterized by low mass and high stiffness, conflicts with the NVH performance, since low noise and vibration levels often require heavy and bulky systems, especially at low frequencies of excitation. The combination of NVH performant solutions and lightweight applications is thus a challenging task.

Recently, locally resonant metamaterials demonstrated to be a valid alternative to the commonly used NVH solutions, linking compact and lightweight design to improved $\mathrm{NVH}$ performance, especially at the lower frequency range [1]. These metamaterials are obtained by including resonant elements to a host structure. The local dynamic interaction between the constituent elements enables them to exhibit stopband behavior in some tunable frequency zones, within which structural wave propagation is inhibited and consequently sound radiation and sound transmission reduced.

In previous publications [2, 3], the authors applied metamaterial solutions for isolating the structural vibrations along a known onedimensional transmission path and investigated different layouts of metamaterial solutions to combine stopbands. In this paper, the potential of metamaterials is investigated in order to isolate vibrations in a structure with multiple transmission paths. The host structure represents part of a vehicle excited by a shock absorber. The resonant elements are added on a small portion of the structure to limit the mass addition. This application case demonstrates the performance of the metamaterials at low frequencies, when multiple transmission paths are available.

After the introduction, the second and the third section of this paper present the application case and the metamaterial solutions design. The stopband region is predicted through a numerical Unit Cell (UC) approach. The fourth section describes the test set-up, followed by the fifth section, in which experimental results are compared to the numerical predictions. Finally, the conclusions end the paper by summing up the findings.

\section{Application case}

The shock absorber is one of the transmission paths that allows vibrations to enter the vehicle. These vibrations can excite body modes of the vehicle, leading to an amplification of the vibration levels. Therefore it would be interesting to isolate the vibration energy upon entering the body. Commonly, the energy is injected at frequencies below $400 \mathrm{~Hz}$, which makes this non-trivial problem an ideal case scenario for locally resonant metamaterials.

In order to investigate the feasibility of the concept of metamaterials for vibration isolation, a simplified set-up of the shock tower of a body in white is made. Its design and dimensions are sketched in Figure 1. The host structure consists of four parts of steel plates, shaped from the same panel. Part (1) is a wide flat plate to ensure model behavior to frequencies below $400 \mathrm{~Hz}$. Part (2) and (3) are welded together to part (1) and (4) at their connection points, forming an angle of $30^{\circ}$. Part (2) and (3) make two separate transfer paths between part (1) and (4).

Page 1 of 7

$03 / 23 / 2018$ 


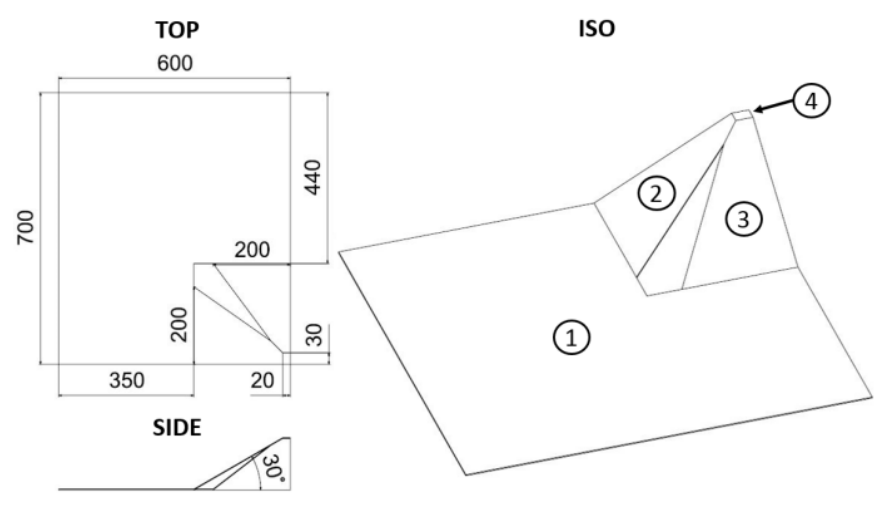

Figure 1-Design of the assembly of the host structure. The dimensions are given in $\mathrm{mm}$.

The thickness and the updated material properties are reported in Table 1. The density is obtained through a weight measurement of the test structures upon assembly. The Young's modulus, Poisson's ratio and structural damping are obtained based on the correlation between the mode shapes retrieved with the Finite Element (FE) model and the experimentally measured mode shapes of a rectangular sample of dimensions 146 x $200 \mathrm{~mm}$, obtained from the same panel from which the host structure is constructed. For the FE model 1851 shell elements are used. For the experimental test the sample is freely suspended and a roving hammer approach and one lightweight accelerometer are used.

Table 1 -Material parameters and thickness of the host structure.

\begin{tabular}{|l|l|}
\hline Young's modulus & $207.4 \mathrm{GPa}$ \\
\hline Poisson's ratio & 0.268 \\
\hline Density & $7881.6 \mathrm{~kg} / \mathrm{m}^{3}$ \\
\hline Structural damping & $0.1 \%$ \\
\hline Thickness & $2.03 \mathrm{~mm}$ \\
\hline
\end{tabular}

\section{Metamaterial solution}

Stopband behavior in locally resonant metamaterials is obtained when the resonant elements are added onto the host structure on a scale smaller than the structural wavelength that propagates in the host structure at the tuned frequency of the resonators [4]. Besides, the net sum of the forces on the host structure exerted by the resonators needs to be non-zero [5].

As in previous work $[2,3]$, the resonant structures are added to the host structure and they have a similar design: a body, which consists of a cantilever beam, a support to connect the resonant structure to the host structure, and an end point mass. The body of the resonator is made by laser cutting the shape from a $6 \mathrm{~mm}$ thickness panel of Polymethylmethacrylate (PMMA). The end point mass is cut from a steel beam and connected together to the body with superglue. The design and picture of the manufactured resonant structure are shown in Figure 2.
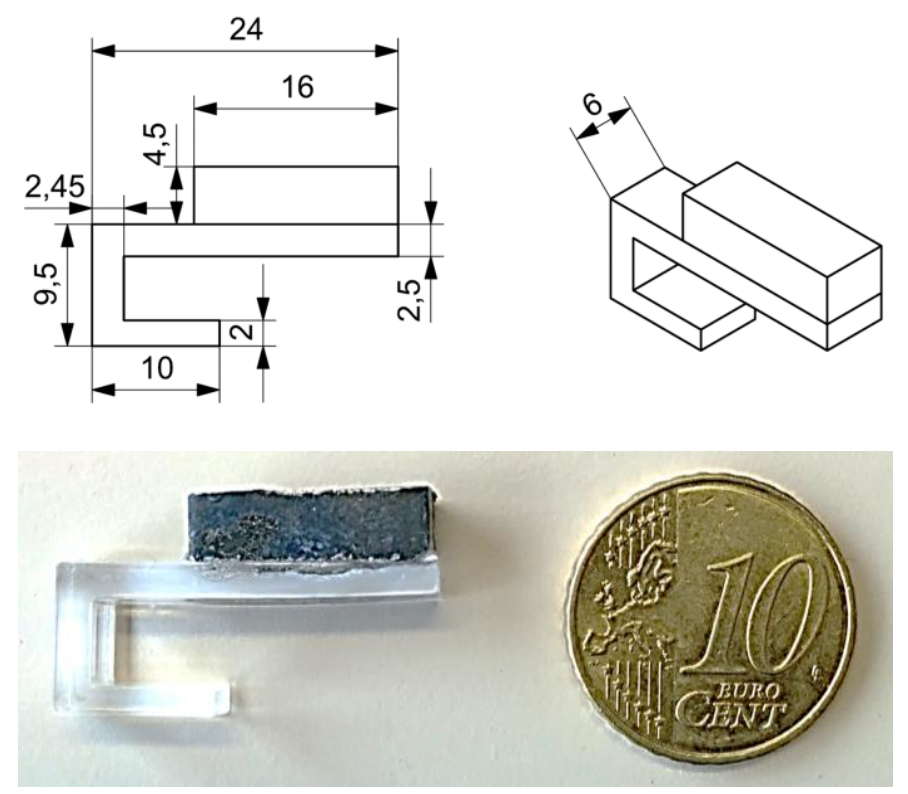

Figure 2 - (Top) Design of the resonant structures. The dimensions are given in $\mathrm{mm}$. (Bottom) Picture of the manufactured resonant structure.

The material properties of the resonators are presented in Table 2. These parameters are retrieved again through correlation of the mode shapes of an FE model and the measured mode shapes of a test sample. In this case, the test sample of the PMMA is a flat plate of dimensions $300 \times 200 \times 6 \mathrm{~mm}$, whereas the test sample for the steel is a beam of dimensions 1000x16x6 mm.

Table 2-Material parameters of the PMMA and steel material for the resonant structures.

\begin{tabular}{|l|l|l|}
\hline & PMMA & Steel \\
\hline Young's modulus & $4.85 \mathrm{GPa}$ & $209.9 \mathrm{GPa}$ \\
\hline Poisson's ratio & 0.31 & 0.298 \\
\hline Density & $1188.4 \mathrm{~kg} / \mathrm{m}^{3}$ & $7685 \mathrm{~kg} / \mathrm{m}^{3}$ \\
\hline Structural damping & $5 \%$ & $1 \%$ \\
\hline
\end{tabular}

The resonant structure is designed to have a resonance frequency at $291 \mathrm{~Hz}$. The FE model used to predict the resonance frequency consists of solid elements and the base of the resonator is clamped. The resonance frequency of the produced resonant structures is measured by gluing the base of the resonant samples on an electrodynamic shaker and measure the velocity on the tip of the end mass by means of a Polytec PSV-500 Scanning Laser Doppler Vibrometer (SLDV). The resonance frequency of the manufactured resonant structures is obtained by measuring 15 resonant structures and calculating the Root Mean Square (RMS) average of those 15 resonance frequencies. The difference between the resonance frequency numerically calculated and experimentally retrieved is less than $1 \%$.

\section{Prediction of the stopband behavior with the FE model}

In this paper, stopband behavior is predicted using UC modelling and by applying Bloch-Floquet boundary conditions. An undamped FE approach is used to model the UC [6,7]. From the UC analysis 
dispersion diagrams are obtained; their derivation and interpretation are discussed by the authors in previous works [1, 4]. Stopbands are identified in the dispersion diagrams as frequency regions where no solution is found, therefore no free wave propagation is possible [8].

To achieve stopband behavior, the resonant structures are added on a regular grid at a distance of $35 \mathrm{~mm}$ in $x$ direction and $20 \mathrm{~mm}$ in $y$ direction. The FE model for the UC analysis consists of 800 linear shell elements for the host structure and 1536 linear solid elements for the resonant structure.

The dispersion diagrams for the UC analyzed are showed in Figure 3. The solution is represented along the irreducible Brillouin contour [9] $\mathrm{O}, \mathrm{A}, \mathrm{B}, \mathrm{C}, \mathrm{O} \rightarrow(0,0),(\pi, 0),(\pi, \pi),(0,0)$, sketched in Figure 4.
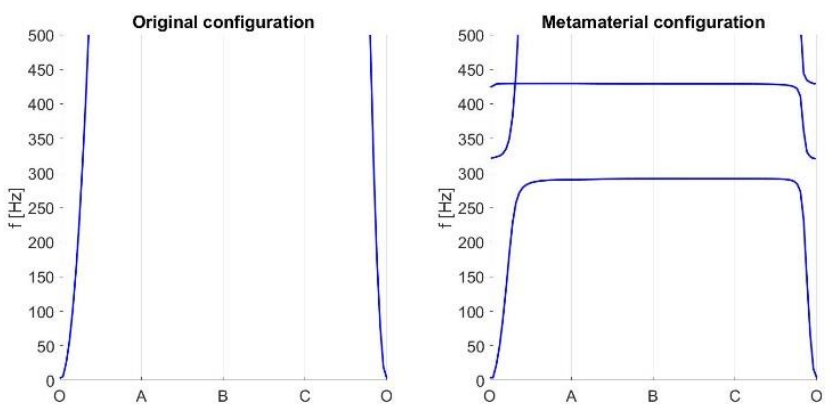

Figure 3 - Dispersion diagrams for the UC without the resonant structure, indicated as original configuration (left), and for the UC with the resonant structure included (right). The horizontal axis represents the irreducible Brillouin contour. The vertical axis represents the frequency.
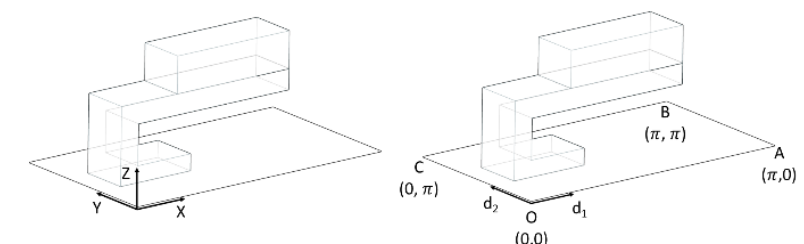

Figure 4 - Sketch of the UC with the resonant element, indicated as metamaterial configuration, in Cartesian coordinates (left) and in the reciprocal wave space (right).

By comparing the dispersion diagram for the UC in original configuration, thus without resonant element in Figure 3 (left), to the dispersion diagram for the UC in metamaterial configuration in Figure 3 (right), a stopband opens in the dispersion diagram for the metamaterial configuration between $291.6 \mathrm{~Hz}$ and $322.6 \mathrm{~Hz}$.

\section{Position of the resonant structures on the host structure}

In this metamaterial application, the resonant structures cover a confined area on the host structure, being different from the previous work $[2,3]$. This choice limits the mass addition to the host structure.

The resonant structures are connected with superglue on part (2) and (3) of the host structure: 44 resonators per part leading to a total of 88 additions. The addition and the orientation of the UC in Figure 4 is shown in Figure 5. The resonant structures are distributed over eight rows, as indicated in Figure 5 by the tags $\mathrm{R} 1$ to R8. Each row contains a different number of resonant structures: starting by two in $\mathrm{R} 1$, an Page 3 of 7 additional resonator is added in each row leading to nine resonant structures in R8.

Each resonant structure weighs about 3.97 grams and adds $33 \%$ of mass on UC level and about $0.06 \%$ of mass on the entire host structure. Therefore, the total added mass on the host structure due to all 88 resonant structures is about $5.3 \%$.

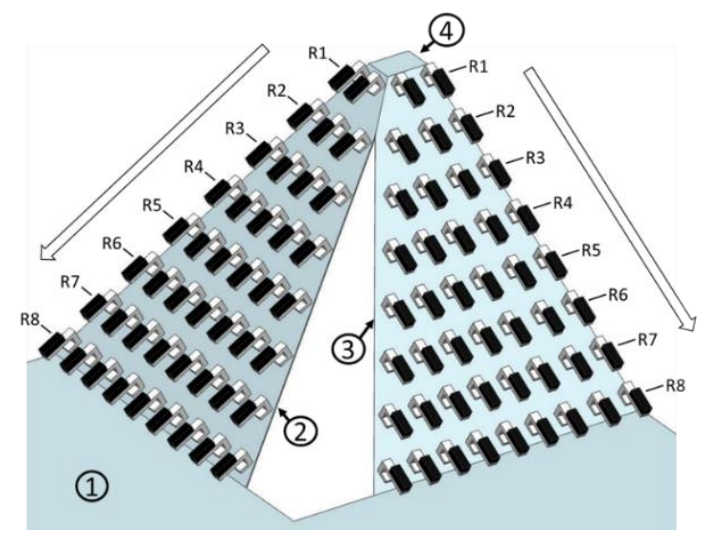

Figure 5 -Position of the resonant structures on the host structure.

\section{Experimental procedure}

This section describes the test set-up and the procedure to measure the effect of the metamaterial additions to the vibration behavior of the setup.

\section{The measurements set-up}

The host structure is freely suspended, as shown in Figure 6, by means of two thin wires connected to part (1). The connection points of the wires on the part (1) of the host structure are at $1 / 3$ and $2 / 3$ of the length of the side.

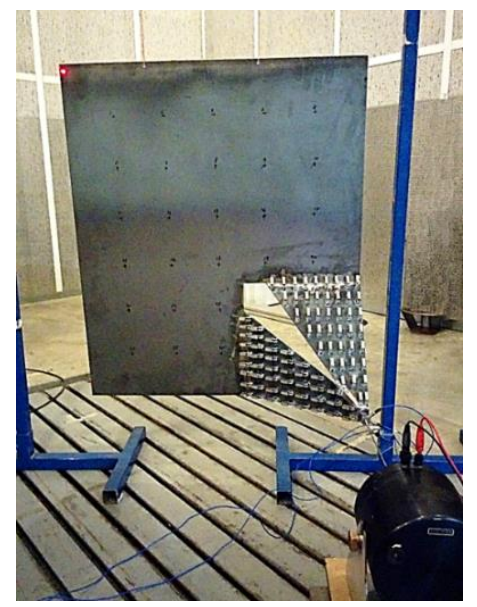

Figure 6 - Picture of the test set-up with the freely suspended metamaterial system and the shaker connected to it.

The shaker excitation is chosen to have better control on the energy injected in the system. A vibration exciter B\&K Type 4809 is connected to the part (4) of the host structure by means of a stinger and an impedance head PCB Model 288D01. 


\section{The force input and the response measurements}

To generate the signal for the excitation and to measure the vibration response of the system, a Polytec PSV-500 SLDV is used.

The signal is sent from the Polytec frontend to the shaker through an amplifier. Random white noise is selected as excitation signal, being representative of a steady state excitation of the shock tower by a regular road profile. Although transient excitation can emulate the shocks encountered by the shock absorber, in this paper only the former is applied. The excitation frequency range is from $75 \mathrm{~Hz}$ to 800 $\mathrm{Hz}$ with a resolution of $0.5 \mathrm{~Hz}$.

The response of the system is evaluated over 67 points located at the free edges of the part (1) of the host structure, as represented in Figure 7. These locations are chosen since, given the free boundary conditions, the highest vibration response is expected at the edges.

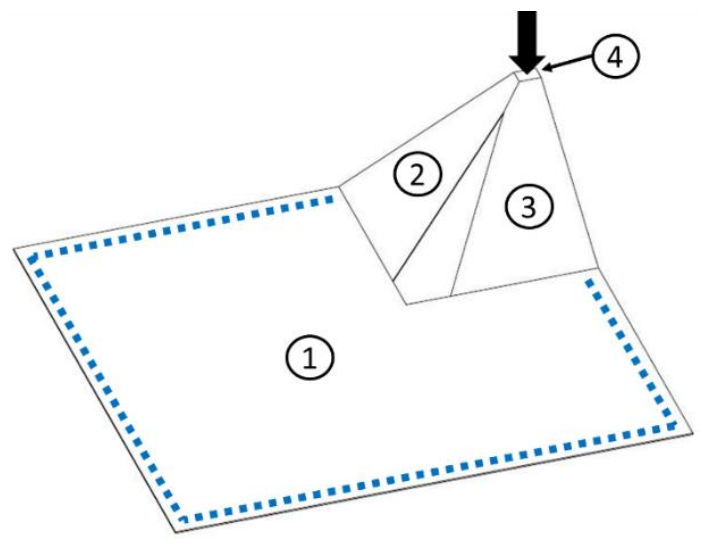

Figure 7 -Representation of the host structure with the force location on part (4) (black arrow) and the measurement points (blue dashed lines).

The amount of averages per response point is set to 15 , which provides a coherence of the Frequency Response Function (FRF) velocity/force above 0.9 in the frequency range of interest. The RMS value of the FRF velocity/force $\left(R M S \frac{v}{F}\right)$, which then represents the amount of energy at the free edges of the system, is calculated per frequency across the response points as expressed by equation (1):

$R M S_{\bar{v}}(\omega)=\sqrt{\frac{1}{N} \sum_{i=1}^{N}\left|\frac{v_{i}}{F}(\omega)\right|^{2}}$,

where $\omega$ is the frequency, $\frac{v_{i}}{F}(\omega)$ indicates the FRF velocity/force per response point and $N$ is the number of the response points.

\section{Measurements results and discussion}

In this section, the measurements results of the tested configurations reported in Table 3 are discussed.
Table 3 - Summary of the tested configurations of the resonant structures on the host structure.

\begin{tabular}{|l|l|}
\hline Configuration & Resonant structures location \\
\hline Original & $/$ \\
\hline A & $\begin{array}{l}\text { Part (2) and (3): two transfer } \\
\text { paths treated }\end{array}$ \\
\hline B & $\begin{array}{l}\text { Part (2): one transfer path } \\
\text { treated }\end{array}$ \\
\hline
\end{tabular}

The measurement results in the graphs are shown between $150 \mathrm{~Hz}$ and $450 \mathrm{~Hz}$ to have a better visualization of the difference in response among the tested configurations around the predicted stopband frequency range.

\section{Original configuration}

The original configuration is used as a reference case. As shown in Figure 8, modes are present in the frequency region around $300 \mathrm{~Hz}$, where the stopband is predicted by the UC analysis.

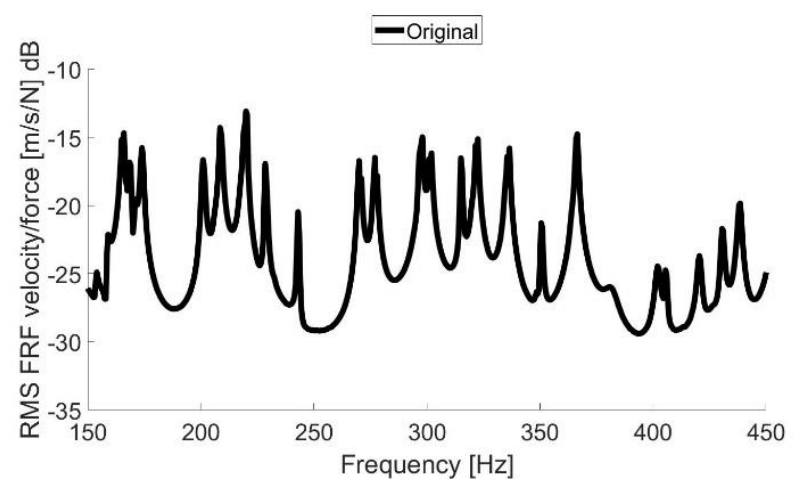

Figure 8-RMS FRF velocity/force of the host structure in the original configuration. The dB reference value is $1 \mathrm{~m} / \mathrm{s} / \mathrm{N}$.

\section{Configuration A}

In configuration $\mathrm{A}$, the resonant structures are added row by row on both the transfer paths: parts (2) and (3). The response of the metamaterial system is measured after every addition of a new row. In Figure 9 only four of these cases are shown for clarity.

Comparing the response of the original configuration to the configuration A with eight rows of resonant structures, an attenuation of the response is noticed inside and around the predicted stopband region. With only one row of resonant structures, the RMS FRF velocity/force levels of the configuration $\mathrm{A}$ and the original configuration are comparable. 


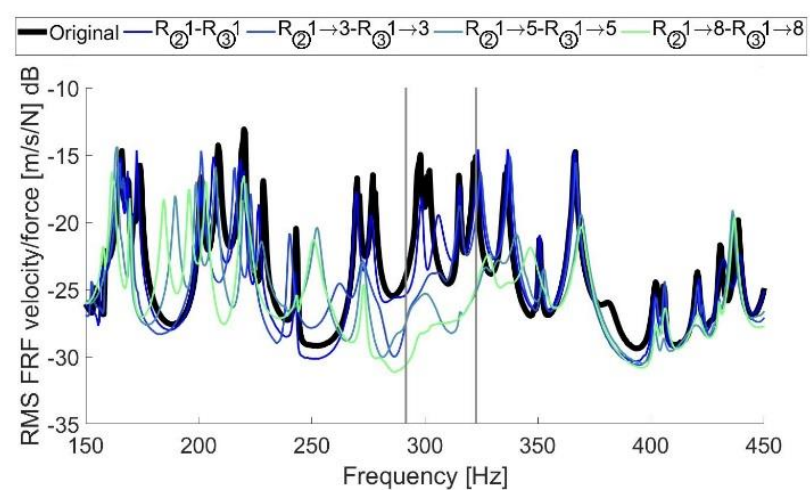

Figure $9-R M S$ FRF velocity/force of the host structure in original configuration and configuration A. The black vertical dotted lines represent the predicted stopband region. The $d B$ reference value is 1 $\mathrm{m} / \mathrm{s} / \mathrm{N}$. The gray vertical lines indicate the predicted stopband frequency range. In the legend, the transfer path treated is indicated by subscripted circled number. The amount of rows added per transfer path is also indicated.

The attenuation of the response becomes more pronounced the more rows of resonant structures are added to the host structure. When five rows are added, the level of the RMS FRF velocity/force of the metamaterial system inside the predicted stopband region is reduced at least $3 \mathrm{~dB}$ for every frequency and in average $6.8 \mathrm{~dB}$ with respect to the original configuration. The mean value of the reduction in $\mathrm{dB}$ scale $\left(A V G_{d B}\right)$ is evaluated as expressed by equations (2) and (3):

$$
\begin{aligned}
& A V G_{d B}=10 \log _{10}\left(\frac{A V G_{\text {bare }}}{d B_{\text {ref }}}\right)-10 \log _{10}\left(\frac{A V G_{M M}}{d B_{\text {ref }}}\right), \\
& A V G_{x}=\frac{1}{N_{\omega_{S B}}} \sum_{j=1}^{N_{\omega_{S B}}} R M S_{\frac{v}{F}}\left(\omega_{S B_{j}}\right),
\end{aligned}
$$

where $A V G_{\text {bare }}$ and $A V G_{M M}$ are the arithmetic mean value across frequency of the RMS FRF velocity/force in amplitude scale. The subscript $x$ in equation (3) indicates either the bare or the metamaterial configuration. These averages are evaluated in the predicted stopband frequency range $\left(\omega_{S B}\right)$ for the original and metamaterial configuration respectively. $N_{\omega_{S B}}$ indicates the number of frequency values in the predicted stopband frequency range. The $\mathrm{dB}$ reference value $\left(d B_{\text {ref }}\right)$ is $1 \mathrm{~m} / \mathrm{s} / \mathrm{N}$.

This improvement in the vibration reduction is achieved by adding only about $2.4 \%$ of mass on the host structure. When all the eight rows of are added, the average reduction inside the predicted stopband is 7.6 $\mathrm{dB}$ with an added mass is about $5.3 \%$.

It can be observed that the frequency region of attenuation extends outside the predicted stopband region. The vibration level of the configuration A with more than four rows of resonators is lower than the original configuration. Starting from $275 \mathrm{~Hz}$ up to $325 \mathrm{~Hz}$ a clear attenuation is obtained in a $50 \mathrm{~Hz}$ frequency band as compared to the predicted $31 \mathrm{~Hz}$-wide stopband. This effect can be attributed to the damping in the resonant structures [10].

Before the predicted stopband frequency range, the resonance frequencies of the metamaterial configuration are strongly shifted to lower frequencies with respect to the bare case, while almost not shift occurs after the predicted stopband frequency range. This effect can be attributed to the dynamic effective mass of the metamaterial system [11]:

- $\quad$ Before the predicted stopband the dynamic effective mass increases to reach its peak at the lower limit of the stopband. The resonance frequencies of the metamaterial configuration are shifted to lower frequencies with respect to the bare configuration because of the added mass effect of the resonant structures on the host structure.

- In the stopband the dynamic effective mass becomes negative and rapidly increases to converge to the mass of the host structure outside the predicted stopband frequency range. The resonance frequencies of the metamaterial configuration are almost not shifted with respect to the bare configuration because the resonant structures do not add additional dynamic mass on to the host structure anymore.

\section{Configuration B}

In configuration $\mathrm{B}$, the resonant structures are added only on one of the transfer paths: part (2). Figure 10 shows the response of the original configuration and the response of the configuration $\mathrm{B}$ with eight rows of resonant structure on part (2) and no resonant structures on part (3).

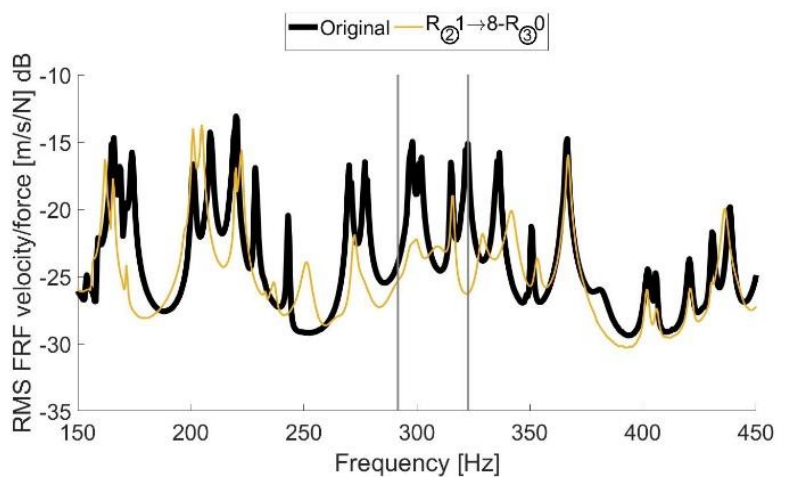

Figure 10 - RMS FRF velocity/force of the host structure in in original configuration and configuration $B$. The black vertical dotted lines represent the predicted stopband region. The $d B$ reference value is 1 $\mathrm{m} / \mathrm{s} / \mathrm{N}$. The gray vertical lines indicate the predicted stopband frequency range. In the legend, the transfer path treated is indicated by subscripted circled number. The amount of rows added per transfer path is also indicated.

Differently from configuration A, configuration $\mathrm{B}$ presents an attenuation of the vibration level as compared to the original configuration around the target frequency of $300 \mathrm{~Hz}$; however, an improvement throughout the whole predicted stopband frequency range is not guaranteed.

\section{Comparison configuration $A$ and configuration $B$}

Figure 11 compares the configuration A and B. The configuration A with 5 rows of resonant structures is kept in Figure 11 because it adds a similar amount of added mass on the host structure as compared to the configuration $\mathrm{B}$. 


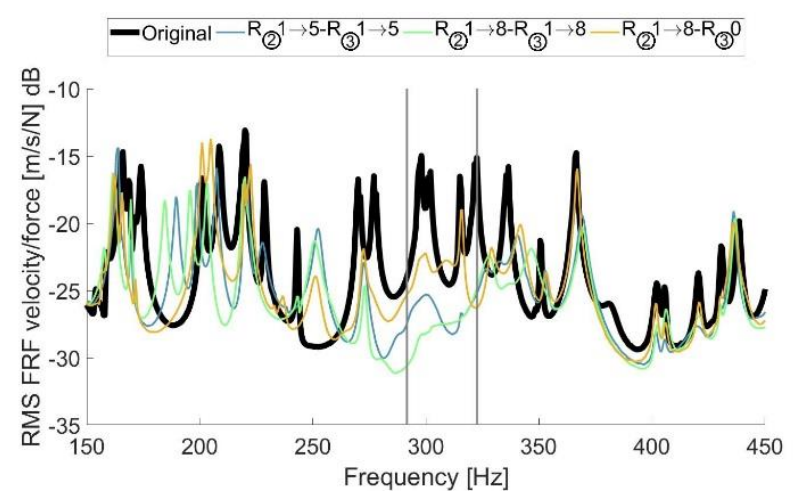

Figure 11 - RMS FRF velocity/force of the host structure in original configuration and configuration A and configuration B. The black vertical dotted lines represent the predicted stopband region. The $d B$ reference value is $1 \mathrm{~m} / \mathrm{s} / \mathrm{N}$. The gray vertical lines indicate the predicted stopband frequency range. In the legend, the transfer path treated is indicated by subscripted circled number. The amount of rows added per transfer path is also indicated.

In configuration $\mathrm{A}$, when the resonant structures are placed on both the transfer paths, the energy propagating from part (4) to part (1) is clearly attenuated. In configuration $\mathrm{B}$, when only one of the transfer path treated with resonant structures, only a small part of the energy is attenuated by the treated transfer path, whereas the rest of the energy can still propagate through the free transfer path.

\section{Summary/Conclusions}

This paper presents a metamaterial application with the purpose of isolating the vibration response in a system with multiple transfer paths in a low frequency range (around $300 \mathrm{~Hz}$ ) and with a low mass and compact volume.

The metamaterial system is realized by applying resonant structures to a small portion of a host structure. The resonant structures are modelled with an FE model and the metamaterial performance is predicted with a UC analysis. The numerical design is experimentally validated.

Test results are carried out in three different configurations: original, configuration A and configuration B.

- Configuration A: both transfer paths are treated with resonant structures. A good agreement between the numerical prediction and the frequency region of attenuation is reached. An average $6.8 \mathrm{~dB}$ of attenuation of the vibration level reduction is obtained inside the predicted stopband region with five rows of resonant structures and $2.4 \%$ of added mass. A further vibration reduction with respect to the bare configuration is reached in a $50 \mathrm{~Hz}$ frequency region centered on $300 \mathrm{~Hz}$.

- Configuration B: only one transfer path is treated with resonant structures. The vibration reduction is limited around $300 \mathrm{~Hz}$.

The comparison of configuration A and B shows the importance of treating both transfer paths to reduce the energy propagating from the force location to the system in which the vibrations need to be reduced.

\section{References}

1. Claeys, C. C., Deckers, E., Pluymers, B., \& Desmet, W. (2016). A lightweight vibro-acoustic metamaterial demonstrator: numerical and experimental investigation. Mechanical Systems and Signal Processing, 70, 853--880. doi:10.1016/j.ymssp.2015.08.029

2. Melo, N. F., Claeys, C., Deckers, E., Pluymers, B., \& Desmet, W. (2016). Dynamic Metamaterials for Structural Stopband Creation. SAE International Journal of Passenger CarsMechanical Systems, 9(2016-01-1791), 1013--1019. doi:doi.org/10.4271/2016-01-1791

3. Claeys, C., Melo, N. F., Van Belle, L., Deckers, E., \& Desmet, W. (2017). Design and validation of metamaterials for multiple structural stop bands in waveguides. Extreme Mechanics Letters, 12, 7--22. doi:10.1016/j.eml.2016.08.005

4. Claeys, C., Vergote, K., Sas, P., \& Desmet, W. (2013). On the potential of tuned resonators to obtain low-frequency vibrational stop bands in periodic panels. Journal of Sound and Vibration, 332(6), 1418--1436. doi:10.1016/j.jsv.2012.09.047

5. Wang, G., Wen, X., Wen, J., Shao, L., \& Liu, Y. (2004). Twodimensional locally resonant phononic crystals with binary structures. Physical review letters, 93(15), 154302. doi:10.1103/PhysRevLett.93.154302

6. Mace, B. R., \& Manconi, E. (2008). Modelling wave propagation in two-dimensional structures using finite element analysis. Journal of Sound and Vibration, 318(4), 884--902. doi:10.1016/j.jsv.2008.04.039

7. Diaz, A. R., Haddow, A. G., \& Ma, L. (2005). Design of bandgap grid structures. Structural and Multidisciplinary Optimization, 29(6), 418--431. doi:10.1007/s00158-004-0497-6

8. Liu, Z., Zhang, X., Mao, Y., Zhu, Y., Yang, Z., Chan, C., . . . Sheng, P. (2000). Locally resonant sonic materials. Science, 289(5485), 1734--1736. doi:10.1126/science.289.5485.1734

9. Brillouin, L. (1946). Wave propagation in periodic structures (Vol. 2nd). McGraw-Hill Book Company.

10. Van Belle, L., Claeys, C., Deckers, E., \& Desmet, W. (2017). On the impact of damping on the dispersion curves of a locally resonant metamaterial: Modelling and experimental validation. Journal of Sound and Vibration, 409, 1--23. doi:10.1016/j.jsv.2017.07.045

11. Nateghi, A., Van Belle, L., Claeys, C., Deckers, E., Pluymers, B. \& Desmet, W. (2017). Wave propagation in locally resonant cylindrically curved metamaterial panels. International Journal of Mechanical Sciences, 127, 73--90. https://doi.org/10.1016/j.ijmecsci.2016.07.003 


\section{Contact Information}

The Research Fund KU Leuven is gratefully acknowledged for its support. This research was also partially supported by Flanders Make, the Strategic Research Centre for the manufacturing industry. The research of E. Deckers is funded by a grant from the Research Foundation - Flanders (FWO).

\section{Definitions/Abbreviations}

AVG

FE
Average (Arithmetic mean)

Finite Element
Frequency Response Function

Noise Vibration Harshness

Polymethylmethacrylate

RMS

Root Mean Square

SLDV

Scanning Laser Doppler Vibrometer

Unit Cell

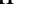

